Research Paper

\title{
Professional Oral Health Care at General Dental Clinic Reduces Postoperative Complications of Head and Neck Free-Flap Reconstruction Surgery
}

\author{
Masatoshi Usubuchi ${ }^{\bowtie}$, Kazuto Matsuura², Takahiro Goto ${ }^{3}$, Yukinori Asada², Takayuki Imai2 ${ }^{2}$, Takenori \\ Ogawa ${ }^{4}$, Kengo Kato ${ }^{4}$, Shigeru Saijo ${ }^{2}$ \\ 1. Department of Dentistry, Miyagi Cancer Center \\ 2. Department of Head and Neck Surgery, Miyagi Cancer Center \\ 3. Department of Plastic and Reconstructive Surgery, Miyagi Cancer Center \\ 4. Department of Otolaryngology-Head and Neck Surgery, Tohoku University Graduate School of Medicine \\ $\square$ Corresponding author: Masatoshi Usubuchi: Department of Dentistry, Miyagi Cancer Center, 47-1, Nodayama, Medeshima-Shiote, Natori, Miyagi Prefecture, \\ 981-1293, Japan. Tel: +81-22-384-3151 extension 8847; Fax: +81-22-381-1174; E-mail: masatoshi-usubuchi@miyagi-pho.jp \\ (C) Ivyspring International Publisher. This is an open access article distributed under the terms of the Creative Commons Attribution (CC BY-NC) license \\ (https://creativecommons.org/licenses/by-nc/4.0/). See http://ivyspring.com/terms for full terms and conditions.
}

Received: 2018.03.26; Accepted: 2018.10.23; Published: 2019.01.01

\begin{abstract}
Background: Extensive resection and free-flap reconstruction surgery has become the standard treatment for locally advanced head and neck cancer. Surgical site infection (SSI) is one of the serious complications of this treatment. This study aimed to investigate the risk factor for onset of SSI, particularly focusing on whether preoperative professional oral health care in cooperation with general dental clinics is effective in reducing the occurrence of SSI.

Methods: From March 2003 to August 2011, 183 patients who underwent head and neck free-flap reconstructive surgery by the same plastic surgeon at Miyagi Cancer Center for Head and Neck Surgery were investigated retrospectively.

Results: Of the 183 patients, 135 and 48 were men and women, respectively, with a mean age of 62 (range, 29-82) years. The tumor was located in the oral cavity $(n=76)$, hypopharynx $(n=55)$, oropharynx $(n=28)$, and others $(n=24)$. Clinical stages were stage I/II in 18 , stage III/IV in 164 patients, and benign tumor in one patient, based on UICC classification. SSI occurred in 66 patients (36.1\%). Based on multivariate analysis, professional oral health care $[P=0.0076$, odds ratio $(O R)=$ $0.39]$ and radiation therapy history $(P=0.0214, O R=2.820)$ were shown as factors that are significantly related to SSI.

Conclusion: This study identified history of radiation therapy as a significant risk factor for SSI from univariate and multivariate analysis and revealed that patients receiving preoperative professional oral health care at general dental clinics reduce the risk of SSI. Preoperative professional oral health care in cooperation with general dental clinics has been shown to reduce SSI of head and neck free-flap reconstructive surgery.
\end{abstract}

Key words: Head and neck tumor, free-flap reconstructive surgery, Surgical site infection, Professional oral health care, Medical collaboration, general dental clinic

\section{Introduction}

Since the 1980s, extensive resection and free-flap reconstruction surgery has become one of the main treatments of locally advanced head and neck tumor, and treatment outcomes have improved [1,2]. However, surgical site infection (SSI) is one of the serious complications after head and neck tumor free-flap reconstructive surgery, accounting for $>40 \%$ of all complications $[3,4,5]$, which may be because the surgery is performed at a surgical site where oropharyngeal bacteria are readily exposed to the 
skin, mouth, and pharynx, classified as class II (clean-contaminated wound area) in the surgical wound classification of the CDC guidelines [6]. SSI causes discomfort and psychological stress, resulting in longer hospital stay and increasing medical expenses. In addition, deterioration of the general condition of the patient, such as nutritional status, not only significantly impairs the patient's quality of life but also delays the start of additional treatment and affects the survival rate [7].

Many risk factors for SSI during head and neck tumor surgery have been reported. Based on the CDC guidelines, risk factors include American Society of Anesthesiologists' Association (ASA) scores, age, nutritional status, smoking, and diabetes [6]. Based on previous multiparameter studies, many risk factors for SSI, such as cervical metastasis, albumin level, hemoglobin level, and oral hygiene status, have been found determined $[8,9,10,11,12]$. However, comparing these definitions for SSI is difficult because are in these studies, and the characteristics of patients (e.g., proportion of cases of free-flap reconstructive surgery) are different.

The oral bacterial count has also been reported to be suppressed by oral health care performed by dental practitioners preoperatively, and it is effective in reducing the incidence of SSI in patients undergoing head and neck cancer reconstructive surgery [13]. During the study period, there was no dental department in our hospital; the head and neck surgery department provided preoperative oral care in cooperation with the general dental clinics in the area for patients undergoing head and neck tumor free-flap reconstruction surgeries from March 2005 onward.

The purpose of this study was to clarify the most important risk factor for SSI after head and neck reconstruction and to identify effective strategy for preventing SSI. In particular, we focused on whether preoperative professional oral care in cooperation with the general dental clinics in the area is effective in reducing the occurrence of SSI.

\section{Patients and methods}

\section{Patient}

From March 2003 to August 2011, 183 patients with head and neck tumor who underwent free-flap reconstructive surgery by the same plastic surgeon at Miyagi Cancer Center Head and Neck Surgery (Natori City, Miyagi Prefecture) were retrospectively investigated. This survey was conducted in accordance with the Declaration of Helsinki with the approval of the Miyagi Cancer Center Ethics Review Committee.

\section{SSI strategy and diagnostic method}

For all patients, the following methods were used:

- Cefazolin or Ampicillin-sulbactam was started 30-60 min before incision and continued for 3 days postoperatively as prophylactic antibacterial treatment.

- The surgical field was disinfected with povidone-iodine.

- After the skin and oropharynx was separated with a flap, the site is washed with $\geq 1000 \mathrm{~mL}$ of physiological saline.

- Sealed drainage was performed.

- After washing the surgical field with physiological saline, the mandibular reconstruction plate or artificial instruments were used.

SSI was judged based on the CDC guidelines [6]. SSI is defined as the presence of at least one of the following symptoms within 30 days postoperatively:

- purulent effluent from superficial incisional wound

- separation of microorganisms from aseptically collected liquid or tissue culture from superficial incision

- if there is at least one sign or symptom of infection, such as pain or tenderness, local swelling, redness, or fever, the surgeon carefully opens the incision surface and the culture of the incision is positive

- the surgeon or assistant doctor judges that the incision surface has SSI

- fistulas defined as the presence of oral or pharyngeal leakage are regarded as SSI regardless of origin.

\section{Factors that were reviewed}

Clinical data were recorded; SSI, patient age, sex, tumor location, clinical stage, life history, medical history, and general condition were recorded. Preoperative intervention included preoperative tracheotomy, head and neck surgery, radiotherapy (defined as a dose of $>40$ Gy in the surgical field), and chemotherapy. Pre- and postoperative factors included mandibular resection, reconstruction, duration of surgery, bleeding volume, and blood transfusion.

The presence of diabetes mellitus was defined as a preoperative diagnosis of borderline or more severe diabetes mellitus. The general conditions were as follows: anemia was defined as a hemoglobin concentration of $<8.5 \mathrm{~g} / \mathrm{dl}$; hypoalbuminemia was defined as a serum albumin level of $<4.0 \mathrm{mg} / \mathrm{dl}$; 
diabetes mellitus was defined as a fasting blood glucose level of $\geq 200 \mathrm{mg} / \mathrm{dl}$; and poor performance status was defined as an American Society of Anesthesiologists Physical Status score $\geq 3$.

\section{Professional Oral health care}

All patients received an explanation about the necessity of professional oral care from doctors and nurses at the visit to the hospital head and neck surgical outpatient and recommended dental examination within a week from the day of surgery. Based on self-determination, patients who visited a general dental clinic underwent oral examination, dental plaque and dental calculus removal, and professional mechanical tooth cleaning, and severe periodontitis and severe caries were extracted. Furthermore, it was guided by dentists and dental hygienists regarding the necessity of oral cleaning and self-care method.

Patients in both groups brushed their teeth and performed self-oral cleaning preoperatively; the oral cavity was cleaned thrice a day by a nurse using a sponge brush immediately postoperatively until oral feeding was resumed. We gradually increased self-care after starting oral feeding.

Statistical analysis was performed using SAS version 9.3 (Cary, NC, USA). We examined the relationship between each variable and SSI using univariate analysis. Categorical data and continuous variables were tested using Fisher exact test and Wilcoxon signed rank test, respectively. Variables with $P<0.05$ in the univariate analysis were subjected to multivariate logistic regression analysis for identifying variables that are independent of SSI. $P<$ 0.05 was considered to be statistically significant.

\section{Results}

\section{Patient characteristics}

Table 1 shows the characteristics of patients. The mean age of patients was 62 (range, 29-82) years. Of the 183 patients, 135 and 48 were men and women, respectively. In total, 66 patients received professional oral health care at the general dental clinic, whereas 117 patients did not. Tumor locations include the oral cavity $(\mathrm{n}=76)$, hypopharynx $(\mathrm{n}=55)$, oropharynx $(\mathrm{n}=$ $28)$, cervical esophagus $(n=7)$, and others $(n=24)$. Clinical stages were stage I/II in 18 patients, stage III/IV in 164 patients, and benign tumor in one patient, according to the UICC classification. The tumor recurred in 10 patients. Free abdominal femoral $(n=23)$, free fibular $(n=6)$, free forearm $(n=9)$, free abdominal $(n=80)$, free jejunal $(n=63)$, Latissimus Myocutaneous $(n=1)$, and Scapular $(n=1)$ flaps were constructed.
Table 1. Patient characteristics

\begin{tabular}{llll}
\hline Characteristic & & Patients & $\mathbf{( \% )}$ \\
\hline Age & $<65$ years & 103 & 56.3 \\
Sex & $\geq 65$ years & 80 & 43.7 \\
& & & \\
\multirow{3}{*}{ Location } & Male & 135 & 73.8 \\
& Female & 48 & 26.2 \\
& & & \\
& Oral cavity & 76 & 41.5 \\
& Hypopharynx & 55 & 30.1 \\
& Mesopharynx & 28 & 15.3 \\
& Cervical esophagus & 7 & 3.8 \\
& Maxilla & 6 & 3.3 \\
& Salivary gland & 6 & 3.3 \\
Performance status & 4 & 2.2 \\
& Larynx & 1 & 0.5 \\
& Thyroid & & \\
& & 56 & 30.6 \\
& 1 & 102 & 55.7 \\
& 2 & 24 & 13.1 \\
& 3 & 1 & 0.5 \\
& 4 & 4 & 2.2 \\
& 0/x/benign & 1 & 0.5 \\
& I & 17 & 9.3 \\
& II & 30 & 16.4 \\
& III & 134 & 73.2 \\
\hline
\end{tabular}

\section{Relationship between risk factor and SSI}

SSI occurred in 66 patients (36.1\%). The incidence of SSI in the group receiving professional oral health care was $24.2 \%(16 / 66)$, whereas that in the non-receiving group was $43.6 \%$ (51/117). Univariate analysis showed significant differences in terms of professional oral health care $(\mathrm{P}=0.011)$ and radiotherapy history $(\mathrm{P}=0.042)$ (Table 2$)$. Multivariate analysis revealed that radiotherapy history increased the risk factor $[\mathrm{P}=0.0214, \mathrm{OR}=2.820]$, and professional oral health care reduced the risk factor $[\mathrm{P}$ $=0.0076$, odds ratio $(\mathrm{OR})=0.39 \mathrm{]}$ (Table 3$)$.

The median duration of surgery and blood loss were 596 (range, 284-1094) $\mathrm{min}$ and 390 (range, 50-1900) mL. Surgical time and blood loss were not significantly associated with SSI (Table 4).

\section{Discussion}

Head and neck surgery, particularly head and neck tumor surgery with microvascular free-flap reconstruction, is the most invasive surgery, and the incidence of SSI is high (range, $36.4 \%-50 \%$ ) $[5,9,10]$. In our study, the incidence of SSI was $36.1 \%$. Majority of our patients have T3 and T4 disease $(89.6 \%, 164 / 183)$ and have more advanced cancer than that reported in other studies. However, the incidence of SSI was similar to that reported in other recent research $[3-5,9,10]$. In our study, univariate analysis and multivariate analysis revealed that a history of radiation therapy increased the risk of SSI, and it was identified that professional oral health care at general dental clinics is a factor that reduces the risk of SSI. 
Table 2. Univariate analysis of preoperative and perioperative risk factors

\begin{tabular}{llll}
\hline Variable & Cases (\%) & $\begin{array}{l}\text { Surgical site } \\
\text { infection (\%) }\end{array}$ & P value \\
\hline Sex Male & 73.8 & 35.6 & 0.8617 \\
Age $\geq 65$ years & 43.7 & 38.8 & 0.5372 \\
Poor ASA-PS (>2) & 13.7 & 44.0 & 0.3791 \\
BMI(kg/m2) $\geq 25$ & 15.9 & 31.0 & 0.6743 \\
Albumin <4.0mg/dl & 30.6 & 35.7 & 1.0000 \\
Diabetes mellitus & 11.5 & 33.3 & 1.0000 \\
Smoking & 68.3 & 36.0 & 1.0000 \\
Alcohol & 36.1 & 68.2 & 0.2225 \\
T-stage (T3+T4) & 73.2 & 38.8 & 0.2270 \\
Clinical stages III + IV & 89.1 & 37.4 & 0.3311 \\
Previous cervical operation & 10.4 & 42.1 & 0.6172 \\
Previous chemotherapy & 16.9 & 45.2 & 0.3052 \\
Previous radiotherapy & 13.7 & 56.0 & $0.0417^{*}$ \\
Professional oral health care & 36.6 & 23.9 & $0.0106^{*}$ \\
Reconstructive procedure & & & \\
Mandibulotomy & 31.7 & 39.7 & 0.5116 \\
Maxillectomy & 7.1 & 53.8 & 0.2300 \\
Bone reconstruction & 4.9 & 33.3 & 1.0000 \\
Bone surgery & 36.1 & 39.4 & 0.5231 \\
Surgery time (>10h) & 51.9 & 33.7 & 0.5390 \\
Blood loss (>600ml) & 10.4 & 42.1 & 0.6172 \\
Blood transfer & 4.9 & 55.6 & 0.2872 \\
Tracheotomy & 93.4 & 36.8 & 0.5414 \\
\hline * P 0.05 & & &
\end{tabular}

Table 3. Results of multiple logistic regression analysis of wound infection

\begin{tabular}{llll}
\hline Variables & Odds ratio & $\begin{array}{l}\text { 95\% confidence } \\
\text { interval }\end{array}$ & P value \\
\hline Professional oral health care & 0.39 & $0.20-0.78$ & $0.0076^{*}$ \\
Previous radiotherapy & 2.82 & $1.17-6.82$ & $0.0214^{*}$ \\
\hline${ }^{*} \mathrm{P}<0.05$ & & &
\end{tabular}

Table 4. Relationship between patient characteristics and risk of surgical site infection (continuous data)

\begin{tabular}{lllll}
\hline Factor & Overall Average & $\begin{array}{l}\text { Average with } \\
\text { SSI }\end{array}$ & $\begin{array}{l}\text { Average } \\
\text { without SSI }\end{array}$ & P value \\
\hline $\begin{array}{l}\text { Body mass index } \\
(\mathrm{kg} / \mathrm{m} 2)\end{array}$ & $21.57 \pm 3.49$ & $22.04 \pm 3.46$ & $21.26 \pm 3.44$ & 0.1502 \\
$\begin{array}{l}\text { Duration of surgery } \\
(\mathrm{min})\end{array}$ & $600 \pm 108$ & $615 \pm 107$ & $592 \pm 108$ & 0.166 \\
$\begin{array}{l}\text { Blood loss (ml) } \\
\text { IStandard deviation. }\end{array}$ & $332.9 \pm 292.7$ & $371.2 \pm 338.9$ & $311.2 \pm 262.3$ & 0.184 \\
\hline
\end{tabular}

Microorganisms in the oral cavity are sources of SSI pathogens [14]. Preoperative professional oral health program significantly reduces wound infection after oral cancer surgery [15]. The results of this study were supported by these reports. There are two features of this research. First, the effectiveness of professional oral health care was proven to be applicable to oral cancer surgery as well as to head and neck tumor free-flap reconstruction surgeries. Second, professional oral health care is not performed only by dentists in the general hospital, but by medical collaboration with the community dental clinic. This is a beneficial result for a hospital with head and neck surgery without dentistry. It is also beneficial for patients to access a local dental office for consultation for treatment to be effective.

The reason why SSI is alleviated by professional oral health care administered preoperatively at the dental clinic is considered to be due to the following features of the oral cavity and pharyngeal region. The oropharynx has complicated structures and organs that are not anatomically uniform. Particularly, the oral cavity has a surface area of approximately 220 $\mathrm{cm}^{2}$ with many niches (tooth, buccal mucosa, tongue, mouth cavity, gums, etc.), and the surface is covered by a biofilm. More than 700 kinds of bacteria inhabit the oral cavity, but only $54 \%$ can be cultured [16]. Bacterial harvesting in the oropharynx requires testing sticks of the surface of the tongue (dorsal tongue), accounting for $12 \%$ of the intraoral area [17]. Therefore, only species of bacterial flora in the oral cavity can be evaluated. This may be one reason for the deviation between the result of bacterial culture of the SSI Wound Department and the result of the oral bacteria test. Saliva covering the oral mucosa contains $10^{9}$ bacteria per $\mathrm{mL}$ [18]. The tooth covers $20 \%$ of the oral area, and the biofilm (dental plaque) attached to the surface contains 1 billion bacteria/mg [19]. Generally, pathogenic bacteria are detected at clinically low levels [20, 21]. However, the number and proportion of pathogenic bacteria increases in pathological conditions [18]. Oral diseases are speculated to occur because of harmful changes in the natural balance of microorganisms [22, 23]. A biofilm is formed several minutes after tooth surface cleaning, and bacterial flora changes as time passes. Oral streptococcus is the main facultative anaerobic bacteria present in the oral cavity. However, without self-care and professional oral health care, it becomes a complex biofilm containing many anaerobic bacteria after 7 days, such as gram-negative bacillus spirochete [24]. They are diffused into the saliva and are speculated to be reservoirs for oropharyngeal region/upper respiratory tract infection. Pathologically increasing oral streptococci will also break down salivary pellicles on the mucosal surface and thereby produce enzymes that expose adhesion receptors to pathogenic bacteria $[25,26]$.

Oral biofilm (dental plaque/dental calculus) removal is impossible with antibiotics [27]. Mechanical destruction and elimination by removal of dental calculus and professional mechanical tooth surface cleaning are the only methods to remove these diseased biofilms [28]. Immediately after 1 or 2 weeks of treatment with this method, the total number of subgingival organisms decreased 10- to 100-fold and the proportions of culturable gram-negative organisms and anaerobic organisms decreased 3- to 4 -fold or more. [29]. These previous findings support 
the rationality of the present study, which demonstrated significantly reduced occurrence of SSI by performing self-care daily and professional oral health care at general dental clinics once every 1 to 7 days before surgery [15].

In this study, radiation therapy history was identified as a risk factor for SSI in univariate and multivariate analyses. In multivariate analysis by Lee et al., radiation therapy history [10] was a risk factor for SSI after head and neck surgeries (OR, 2.85). Radiation has been shown to have pathophysiological effect, poor wound healing, and delayed infection [30], which are consistent with our results.

Hypoalbuminemia is the evaluation item that determines whether the nutritional state is a risk factor. Nutritional status is believed to be related to poor healing of tissues, decreased collagen synthesis, and granuloma formation on surgical wounds. In this study, no significant relationship was found between albumin and hemoglobin levels. Accurately determining the nutritional status was impossible because the examination was performed 1-14 days preoperatively, with the half-life of blood albumin at 14-20 days, and dehydration due to difficulty in oral ingestion.

This research has limitations. First, it is an observational research conducted at a single facility. In addition, the preoperative dental condition is not included in the evaluation items. This is because information on the dental condition from the requested dental medical institution could not be obtained. The presence of tooth defects, such as dentures [15], has been reported to be a risk factor for SSI in univariate analysis, but the preoperative dental condition of patients with head and neck tumor is not a serious risk factor for SSI [31]. Therefore, we speculate that quantitatively evaluating and analyzing the condition of the patient's teeth are difficult because each patient may show various dental conditions. Currently, there are methods for quantitatively evaluating various oral hygiene/dental conditions (e.g., OAG [32] and OHAT [33], serum levels of blood-related periodontal disease bacteria [34], and antibody titer). Considering cost effectiveness, prospectively considering whether these are effective as evaluation items is necessary.

In conclusion, this study indicated that preoperative professional oral health care in cooperation with general dental clinics reduces the SSI of head and neck free-flap reconstructive surgery.

\section{Competing Interests}

The authors have declared that no competing interest exists.

\section{References}

1. Panje WR, Bardach J, Krause CJ. Reconstruction of the oral cavity with a free flap. Plast Reconstr Surg. 1976; 58: 415-8.

2. Hayashi R, Ebihara S. The treatment of locally advanced tongue cancer. Otologia Fukuoka. 2001; 47: 51-5.

3. Karakida K, Aoki T, Ota Y, et al. Analysis of risk factors for surgical-site infections in 276 oral cancer surgeries with microvascular free-flap reconstructions at a single university hospital. J Infect Chemother. 2010; 16: 334-9.

4. Liu SA, Wong YK, Poon CK, et al. Risk factors for wound infection after surgery in primary oral cavity cancer patients. Laryngoscope. 2007; 117: 166-71.

5. Penel N, Fournier C, Lefebvre D, et al. Multivariate analysis of risk factors for wound infection in head and neck squamous cell carcinoma surgery with opening of mucosa. Study of 260 surgical procedures. Oral Oncol. 2005; 41: 294-303.

6. Berríos-Torres SI, Umscheid CA, Bratzler DW, et al. Healthcare Infection Control Practices Advisory Committee. Centers for Disease Control and Prevention Guideline for the Prevention of Surgical Site Infection, 2017. JAMA Surg. 2017; 152: 784-91.

7. de Melo GM, Ribeiro KC, Kowalski LP, et al. Risk factors for postoperative complications in oral cancer and their prognostic implications. Arch Otolaryngol Head Neck Surg. 2001; 127: 828-33.

8. Ogihara H, Takeuchi $\mathrm{K}$, Majima Y. Risk factors of postoperative infection in head and neck surgery. Auris Nasus Larynx. 2009; 36: 457-60.

9. Lee DH, Kim SY, Nam SY, et al. Risk factors of surgical site infection in patients undergoing major oncological surgery for head and neck cancer. Oral Oncol. 2011; 47: 528-31.

10. Cunha TF, Soares Melancia TA, Zagalo Fernandes Ribeiro CM, et al. Risk factors for surgical site infection in cervico-facial oncological surgery. J Craniomaxillofac Surg. 2012; 40: 443-8.

11. Cannon RB, Houlton JJ, Mendez E, Futran ND. Methods to reduce postoperative surgical site infections after head and neck oncology surgery. Lancet Oncol. 2017; 18: e405-13.

12. Harréus U. Surgical errors and risks - the head and neck cancer patient. GMS Curr Top Otorhinolaryngol Head Neck Surg. 2013; 12: Doc04

13. Ota Y. Research on actual condition of intraoral complications by cancer treatment and establishment of its prevention method. Ministry of Health, Labor and Welfare Cancer Research Grant Project. 2003;:15-23. http://crdb.ncc.go.jp/search/DRTV050.action?rpno=012006002700000 (in Japanese).

14. Matsunaga K, Oobu K, Yoshikawa H, et al. A clinical study of postoperative infection in oral and maxillofacial surgery. J Jpn Stomatol Soc. 2002; 51: 62-8. (in Japanese)

15. Sato J, Goto J, Harahashi A, et al. Oral health care reduces the risk of postoperative surgical site infection in inpatients with oral squamous cell carcinoma. Support Care Cancer. 2011; 19: 409-16.

16. Human Oral Microbiome Database (HOMD). 2016. Accessed April 2016. http://www.homd.org/

17. Schlagenhauf $\mathrm{U}$, Pommerencke $\mathrm{K}$, Weiger R. Influence of toothbrushing, eating and smoking on Dentocult SM Strip mutans test scores. Oral Microbiol Immunol. 1995; 10: 98-101.

18. Marsh PD. Dental plaque: biological significance of a biofilm and community life-style. J Clin Periodontol. 2005; 32: 7-15.

19. Collins LM, Dawes C. The surface area of the adult human mouth and thickness of the salivary film covering the teeth and oral mucosa. J Dent Res. 1987; 66: 1300-2.

20. Marsh PD, Head DA, Devine DA. Ecological approaches to oral biofilms: control without killing. Caries Res. 2015; 49 (Suppl 1):46-54.

21. Socransky SS, Haffajee AD. Periodontal microbial ecology. Periodontol 2000. 2005; 38: 135-87.

22. Durand ML, Yarlagadda BB, Rich DL, et al. The time course and microbiology of surgical site infections after head and neck free flap surgery. Laryngoscope. 2015; 125: 1084-9.

23. Kilian M, Chapple IL, Hannig M, et al. The oral microbiome-an update for oral healthcare professionals. Br Dent J. 2016; 221: 657-66.

24. Ritz HL. Microbial population shifts in developing human dental plaque. Arch Oral Biol. 1967; 12: 1561-8.

25. Scannapieco FA. Role of oral bacteria in respiratory infection. J Periodontol. 1999; 70: 793-802.

26. Benedek-Spät E, Di Felice R, Andersen E, et al. In vitro release of elastase from human blood and gingival crevicular neutrophils. Arch Oral Biol. 1991; 36: 507-10.

27. Costerton JW, Lewandowski Z, Caldwell DE, et al. Microbial biofilms. Ann Rev Microbiol. 1995; 49: 711-45.

28. Arweiler NB, Netuschil L. The Oral Microbiota. Adv Exp Med Biol. 2016; 902: 45-60.

29. Slots J, Mashimo P, Levine MJ, et al. Microbiological and clinical effects of a single course of periodontal scaling and root planing, and of adjunctive tetracycline therapy. J Periodontol. 1979; 50: 495-509.

30. Dormand EL, Banwell PE, Goodacre TE. Radiotherapy and wound healing. Int Wound J. 2005; 2: 112-27 
31. Penel N, Lefebvre D, Fournier C, et al. Risk factors for wound infection in head and neck cancer surgery: a prospective study. Head Neck. 2001; 23: $447-55$.

32. Eilers J, Berger AM, Petersen MC. Development, testing, and application of the oral assessment guide. Oncol Nurs Forum. 1988; 15: 325-30.

33. Chalmers JM, King PL, Spencer AJ, et al. The oral health assessment tool--validity and reliability. Aust Dent J. 2005; 50: 191-9.

34. Dye BA, Herrera-Abreu M, Lerche-Sehm J, et al. Serum antibodies to periodontal bacteria as diagnostic markers of periodontitis. J Periodontol. 2009; 80: 634-47. 